\title{
Management of intramural hematoma of the ascending aorta: Still room for debate
}

Thoralf M. Sundt, MD

See related article on page 918.
From the Mayo Clinic and Foundation, Rochester, Minn.

Received for publication April 30, 2002; accepted for publication May 15, 2002.

Address for reprints: Thoralf M. Sundt, MD, Mayo Clinic and Foundation, 200 First St SW, Rochester, MN 55905.

J Thorac Cardiovasc Surg 2002;124:894-5

Copyright $\odot 2002$ by The American Association for Thoracic Surgery

$0022-5223 / 2002 \$ 35.00+0 \quad \mathbf{1 2 / 1 / 1 2 6 5 6 6}$

doi: $10.1067 / \mathrm{mtc} .2002 .126566$

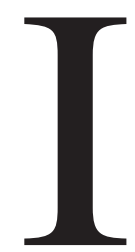

$\mathrm{n}$ this issue of the Journal, Moizumi and colleagues ${ }^{1}$ from Sendai City Medical Center and Tohoku University present a thought-provoking analysis of their experience with what would be considered by most Western surgeons an iconoclastic approach to the management of intramural hematoma of the ascending aorta (IMH). Applying an algorithm of expectant (I would not call it conservative) therapy to 33 patients with this condition during a 9-year interval, these authors have acquired what is to my knowledge the largest reported single-center experience with this uncommon entity. Their results are excellent. They challenge us to rethink our approach to this condition.

In the authors' series, only patients with cardiac tamponade, impending rupture (described as hemodynamic instability or persistent pain), rupture, or progression of the IMH underwent surgery. All others were treated medically with aggressive antihypertensive therapy and bed rest. Nine of 33 patients underwent early surgery with what can only be considered a highly acceptable mortality rate of $11 \%$, given that 5 of the 9 patients were in shock on admission and 3 underwent total arch replacement. Early mortality in the medical group was also low at 5\% ( $P$ not significant). During the follow-up interval, 8 additional patients underwent surgery for progression to frank dissection or for increasing aortic dilatation. Survivals at 1 and 2 years were similar in the two groups. The authors concluded that expectant management with aggressive medical therapy and serial imaging studies, with surgical intervention reserved for those with clinical or radiologic evidence of progression, yields satisfactory results.

"It is hard to argue with success," as one of my mentors is fond of saying, but do these data indicate that medical management is the optimal approach to IMH? Do they indicate equivalent—if not superior-results with medical therapy? Or do they conversely demonstrate remarkably low surgical risk despite the systematic selection of only the sickest patients for surgery?

This study is not a comparison of treatment modalities. That would require random assignment to two groups with a head-to-head comparison. To bicker over surgery versus medicine would be to miss the mark. The issue here is much like the debate regarding coronary angioplasty versus coronary artery bypass: it is the validity and safety of a treatment algorithm that attempts triage of individual patients to their most appropriate therapy, sparing unnecessary risk to those for whom less aggressive management will suffice without withholding such therapy from those who require surgery.

The central message here is not that medical management is preferable but rather that, in selected cases, it is reasonable. These data serve to open the dialog. Previous studies from Western countries have demonstrated rather consistently that IMHs behave differently depending on location-ascending versus descending aorta. A study of IMH reported in 1993 by Robbins and coworkers ${ }^{2}$ suggested a particularly poor prognosis of the condition when it involves the ascending aorta, although the experience was quite small. Of only 3 patients in whom the ascending aorta and arch were involved, 1 survived hospitalization but had late progression to aneurysm requiring surgery, a second had rupture in hospital, and the third underwent surgery on an acute basis. These authors suggested that because of the poor prognosis associated with this condition, early surgery should be considered for type A IMH. This recommendation was widely adopted, and just 2 years thereafter O'Gara and 
DeSanctis ${ }^{3}$ argued that "distinctions between IMH and classic aortic dissection are clinically artificial and should be abandoned so as not to delay appropriate treatment ... patients with aortic IMH should be managed with the same empirical guidelines that have been successfully adopted for patients with classic dissection. Specifically, involvement of the ascending aorta should prompt urgent surgical repair." Since then a number of other small series have supported this notion. A meta-analysis of these studies recently reported by Maraj and associates ${ }^{4}$ identified 81 cases of IMH involving the ascending aorta. The mortality rate among those treated surgically was $14 \%$, whereas that among those treated medically was $36 \%$. The obvious conclusion is that surgical management carries a lower risk than does medical therapy.

The difficulty, of course, is that the conclusions the one can draw from such retrospective studies are significantly limited by the potential impact of selection bias. Were moribund patients refused surgery, driving up the mortality rate in the medical arm? Or were they sent to surgery in desperation for lack of any other option? This problem makes studies such as the one reported in this issue of the Journal by Moizumi and colleagues ${ }^{1}$ vitally important in defining the appropriate management of this condition.

It is now up to the reader to ask the practical questionsdoes this approach make sense to me, and how can I use these results to guide my practice? Can surgery be reasonably delayed in high-risk patients? What of low-risk patients? Of 24 patients initially managed medically, 6 patients ultimately had frank dissection develop, including 2 in whom the IMH initially resolved radiographically. Localized IMH is a very nearly curable disease, so is progres- sion to generalized dissection with the chronic risk of aneurysmal dilatation of the remaining aorta tolerable? And is a hospital stay measured in weeks acceptable, not only to payors but to patients? In an era of next-day (if not sameday) delivery of health care, will patients tolerate the sword of Damocles or the surgeon's scalpel?

It seems likely that IMH is a heterogeneous condition, and its behavior may well depend on a variety of factors including its relationship to other aortic conditions, such as penetrating atherosclerotic ulcer, medial degeneration, or underlying genetic factors affecting connective tissue integrity. Indeed, the disparate results reported from Western centers as compared with this and other centers in Japan have suggested to some that the processes are fundamentally different. Perhaps it is the genetic background that is different. We are indebted to the authors and to the editor for rekindling a controversy that stimulates us to think more deeply about this uncommon but intriguing clinical entity and for challenging us to make our practice evidence based rather than anecdote driven.

\section{References}

1. Moizumi Y, Komatsu T, Motoyoshi N, Tabayasi K. Management of patients with intramural hematoma involving the ascending aorta. J Thorac Cardiovasc Surg. 2002;124:918-24.

2. Robbins RC, McManus RP, Mitchell RS, Latter DR, Moon MR, Olinger GN, et al. Management of patients with intramural hematoma of the thoracic aorta. Circulation. 1993;88(5 Pt 2):II-1-10.

3. O'Gara PT, DeSanctis RW. Acute aortic dissection and its variants: toward a common diagnostic and therapeutic approach. Circulation. 1995;92:1376-8.

4. Maraj R, Rerkpattanapipat P, Jacobs LE, Makornwattana P, Kotler MN. Meta-analysis of 143 reported cases of aortic intramural hematoma. Am J Cardiol. 2000;86:664-8. 